\title{
Reconstruction of Calmodulin Single-Molecule FRET States, Dye-Interactions, and CaMKII Peptide Binding by MultiNest and Classic Maximum Entropy
}

\author{
Matthew S. DeVore ${ }^{\mathrm{a}}$, Stephen F. Gull ${ }^{\mathrm{b}}$, and Carey K. Johnson ${ }^{\mathrm{a}, \dagger}$ \\ aDepartment of Chemistry, University of Kansas, Lawrence, Kansas, 66045, United States \\ ${ }^{b}$ Astrophysics Group, Department of Physics, Cambridge University, Cambridge CB3 OHE, United \\ Kingdom
}

\begin{abstract}
We analyze single molecule FRET burst measurements using Bayesian nested sampling. The MultiNest algorithm produces accurate FRET efficiency distributions from single-molecule data. FRET efficiency distributions recovered by MultiNest and classic maximum entropy are compared for simulated data and for calmodulin labeled at residues 44 and 117. MultiNest compares favorably with maximum entropy analysis for simulated data, judged by the Bayesian evidence. FRET efficiency distributions recovered for calmodulin labeled with two different FRET dye pairs depended on the dye pair and changed upon $\mathrm{Ca}^{2+}$ binding. We also looked at the FRET efficiency distributions of calmodulin bound to the calcium/calmodulin dependent protein kinase II (CaMKII) binding domain. For both dye pairs, the FRET efficiency distribution collapsed to a single peak in the case of calmodulin bound to the CaMKII peptide. These measurements strongly suggest that consideration of dye-protein interactions is crucial in forming an accurate picture of protein conformations from FRET data.
\end{abstract}

\section{Keywords}

calmodulin; FRET; single-molecule fluorescence; fluorescence dyes; Bayesian analysis; nested sampling; maximum entropy; calcium/calmodulin dependent protein kinase II

\section{Introduction}

Single-molecule measurements are an increasingly standard technique in the biophysicist s toolbox. In particular, single-molecule Förster resonance energy transfer (sm-FRET) has been applied to investigate the conformational structure and dynamics of proteins, DNA, and RNA[1-4]. The key to obtaining useful sm-FRET data is a well behaved dye pair. A fluorescent energy donor dye and an energy acceptor must be incorporated into the molecule(s) of interest. These dyes, while attached to biomolecules, should reorient relative to each other on a time scale faster than the FRET efficiency transfer [5]. The dyes should not interact with the parent molecule and, if they become spatially close to one another, they

(C) 2012 Elsevier B.V. All rights reserved.

Corresponding author: Carey K. Johnson, phone: 785-864-4219; fax: 785-864-5396; ckjohnson@ku.edu; address: Department of Chemistry, Malott Hall, University of Kansas, Lawrence, KS 66044 USA.

Publisher's Disclaimer: This is a PDF file of an unedited manuscript that has been accepted for publication. As a service to our customers we are providing this early version of the manuscript. The manuscript will undergo copyediting, typesetting, and review of the resulting proof before it is published in its final citable form. Please note that during the production process errors may be discovered which could affect the content, and all legal disclaimers that apply to the journal pertain. 
should not interact with each other. The nature of molecular interactions makes these criteria hard to achieve. If dye interactions are well characterized, useful results may nevertheless be derived from the data. Ultimately, the reliability of FRET results should be checked using multiple dye pairs.

Single-molecule burst measurements are a powerful sm-FRET method [6-8]. In these measurements, a dilute solution is placed on a confocal microscope. As single molecules diffuse through a laser focus, fluorescence photons are emitted. The time of arrival of each photon is detected by photodiodes. When a plot of photon arrivals is made, bursts of photons are seen which give the measurement its name; see Figure 1. Burst measurements allow tens of thousands of single molecules to be probed in a short time. Burst measurements can be made in biologically realistic conditions, with appropriate $\mathrm{pH}$ and ionic strength. The measurements do not require immobilization of the molecules by, for example, directly tethering them to a surface or encapsulating them in vesicles or gels. This facilitates investigation of the conformations, kinetics and binding constants with minimal change to the native environment of the biomolecule.

Significant improvements have been made since the first sm-FRET burst measurements. Alternating and pulsed lasers have been used to investigate dye bleaching, fluorescence lifetimes, quantum yield inhomogeneities, anisotropies, and dye-biomolecule stoichiometry [9-13]. Bayesian approaches [14-22] and complementary maximum entropy methods [2325] have been implemented for single-molecule data analysis.

We studied FRET efficiency distributions obtained from dye labeled calmodulin, using smFRET bursts. Calmodulin is a small $16.7 \mathrm{kDa}$ protein found in all eukaryotic cells. It is an integral part of the cell s calcium signaling pathway. Calmodulin binds up to four $\mathrm{Ca}^{2+}$ ions, with high affinities. It activates and deactivates target proteins in accordance with its $\mathrm{Ca}^{2+}$ occupancy. Its structure is relatively simple, consisting of two domains that each contain two EF-hand motifs; see Figure 2 [26,27]. Calmodulin exists in both compact and elongated conformations [27-31].

One of the enzymes that binds calmodulin is calcium/calmodulin dependent protein kinase II (CaMKII)[32]. CaMKII is activated by $\mathrm{Ca}^{2+}$-calmodulin, and phosphorylates its substrates so as to regulate a variety of cellular functions [33]. Isoforms of CaMKII have been found in the heart, brain, lung, pancreas, liver, and skeletal muscle (see [34] and references therein). The structure of calmodulin that is bound to a peptide from the binding domain of CaMKII has been determined [35] (see Figure 2B). The FRET distributions stemming from calmodulin bound to the CaMKII binding domain were investigated in this work.

We attached a fluorescent dye to each domain of calmodulin; see Figure 2. Upon excitation of the donor dye, FRET can take place between the dyes. Single-molecule burst FRET measurements were made on freely diffusing molecules. The resulting FRET data were then analyzed via a Bayesian nested sampling approach. This analysis has not been performed before and its results were compared to those obtained using the classic maximum entropy method (cMEM) for sm-FRET bursts [22]. Both methods were applied to analyze FRET distributions for calmodulin under conditions of high and low $\mathrm{Ca}^{2+}$ and also in the presence and absence of the CaMKII binding-domain peptide. We compared the two methods of analysis and, below, we discuss the role of dye-protein interactions in the FRET distributions. 


\section{Methods}

\section{Sample preparation}

Calmodulin was mutated at sites 44 and 117, changing the native threonine residues to cysteines (CaM-44-117). The protein was expressed in E. coli. The cells were induced with $0.4 \mathrm{mM}$ IPTG at an O.D. of 0.7. Then they were pelleted by centrifuging and re-suspended in TRIS or MOPS buffer. The cells were lysed by adding $150 \mu \mathrm{g} / \mathrm{ml}$ lysozyme, with incubation at $37^{\circ} \mathrm{C}$, and sonicated three times in an ice bath for 30 seconds. Cell debris was separated by centrifuging at $19,500 \mathrm{rpm}$ for 30 minutes at $4{ }^{\circ} \mathrm{C}$. $\mathrm{CaCl}_{2}$ was added to the sample to a final concentration of $5 \mathrm{mM}$. CaM-44-117 was purified on an Äkta FPLC with a $25 \mathrm{ml}$ bed of Phenylsepharose 6 Fast Flow (high-sub) column (XK 16/20 GE Healthcare). Before sample loading, the column was equilibrated with $50 \mathrm{mM}$ TRIS, $1 \mathrm{mM} \mathrm{CaCl}_{2}, \mathrm{pH}$ 7.5. The column was then washed with two column volumes of $50 \mathrm{mM}$ TRIS, $1 \mathrm{mM} \mathrm{CaCl}_{2}$, $\mathrm{pH} 7.5$, followed by three column volumes of $50 \mathrm{mM}$ TRIS, $1 \mathrm{mM} \mathrm{CaCl}_{2}, 500 \mathrm{mMNaCl}$, $\mathrm{pH} 7.5$, and three more column volumes of $50 \mathrm{mM}$ TRIS, $1 \mathrm{mM} \mathrm{CaCl}_{2}, \mathrm{pH} 7.5$. CaM-44-117 was eluted from the column with $10 \mathrm{mM}$ TRIS, $10 \mathrm{mM}$ EDTA, pH 7.5. The elution peaks containing CaM-44-117 were further purified on a Superdex size exclusion column (GE Healthcare) in $10 \mathrm{mM}$ HEPES, $0.1 \mathrm{M} \mathrm{KCl}, 1.0 \mathrm{mM} \mathrm{MgCl}_{2}, 0.1 \mathrm{mM} \mathrm{CaCl}_{2}, \mathrm{pH}$ 7.4. The protein was aliquoted into $2.1 \mathrm{mg}$ portions and stored at $-80^{\circ} \mathrm{C}$.

The cysteines were labeled with fluorescent FRET dye pairs by maleimide reaction chemistry, as described previously [36]. The FRET donor dye was Alexa Fluor 488 C5 maleimide (AF488) (Molecular Probes). The acceptor was either Alexa Flour 594 C5 maleimide (AF594) or Texas Red C2 maleimide (TR) (Molecular Probes). Free dye was separated from dye-labeled protein by means of size exclusion chromatography (G-25 Sephadex fine, GE Healthcare Life Sciences). CaM-44-117 labeled with two donor or two acceptor dyes was separated from the FRET species containing one donor and one acceptor dye by C18 reverse phase HPLC (Jupiter 3u C18 300A 250×4.6mm column, Phenomenex). The HPLC solvent was exchanged with either $10 \mathrm{mM}$ HEPES or $30 \mathrm{mM}$ HEPES, $0.1 \mathrm{M}$ $\mathrm{KCl}, 1.0 \mathrm{mM} \mathrm{MgCl}_{2}, 0.1 \mathrm{mM} \mathrm{CaCl}_{2}, \mathrm{pH} 7.4$, and was stored at $-80 \mathrm{C}$. The resulting samples were CaM-44-117 labeled with AF488 and AF594 (CaM-44-117-AF488-AF594), or CaM-44-117 labeled with AF488 and TR (CaM-44-117-AF488-TR).

Calmodulin samples were diluted to $15-40 \mathrm{pM}$ for sm-FRET burst measurement. The experiments were performed in $30 \mathrm{mM}$ HEPES, $0.1 \mathrm{M} \mathrm{KCl}, 1.0 \mathrm{mM} \mathrm{MgCl}_{2}, 0.1 \mathrm{mM} \mathrm{CaCl}_{2}$, $\mathrm{pH}$ 7.4, which will be called high $\mathrm{Ca}^{2+}$ buffer, and $30 \mathrm{mM}$ HEPES, $0.1 \mathrm{M} \mathrm{KCl}, 1.0 \mathrm{mM}$ $\mathrm{MgCl}_{2}, 3.0 \mathrm{mM}$ EGTA, pH 7.4 (low $\mathrm{Ca}^{2+}$ buffer). For measurements of dye-labeled calmodulin in the presence of CaMKII peptide, the peptide was added to the solution up to a final concentration of $1.1 \mu \mathrm{M}$. Calcium/calmodulin dependent protein kinase II peptide fragment 290-309 was purchased from Sigma Aldrich. The sample was dissolved in high $\mathrm{Ca}^{2+}$ buffer. The peptide was stored at $-20{ }^{\circ} \mathrm{C}$ prior to use.

\section{Single molecule microscope}

Burst measurements were made with an inverted Nikon TE 2000 fluorescence microscope system, as described previously [37]. Donor dye excitation was performed with a $488 \mathrm{~nm}$ argon ion laser (2201-20SL, JDS Uniphase, San Jose, CA) of power $25 \mu \mathrm{W}$ measured ahead of the microscope. The laser beam was transformed to circular polarization by a consecutive linear polarizer and quarter-wave plate, and was directed into the microscope by a 500DCXR dichroic beam splitter (Chroma). To spectrally separate the donor and acceptor emission bands, we used emission filters, ET535/50m and HQ 650/100m-2p, and a dichroic beam splitter, 565 DCLP (Chroma Technology Corp., Bellows Falls, VT). 
Samples were placed in petri dishes fitted with a bottom cover slip (MatTek Corp., Ashland, MA). Sample cell surfaces were coated with bovine serum albumin (BSA) prior to the experiments; BSA minimizes sample loss due to surface adsorption. Multiple data sets from two separate days were combined to give a total of approximately 16 hours of collection for each sample type.

\section{Simulated Data}

Simulated data were used to test the MultiNest fitting model. Random numbers were generated according to the photon distribution analysis (PDA) [38]. Two Gaussian distributions were used to simulate $P(E)$. The fluorescence dimension was Poisson distributed, with an average background of 20 photons for each simulation. Background noise was added to the simulated data. The background noise was Poisson distributed with a mean of 0.3 counts in the donor channel and 0.2 in the acceptor channel. A detailed description of the simulated data algorithm has been published elsewhere [22].

\section{Theory}

Bayesian analysis is encapsulated in Bayes theorem for relating probabilities,

$$
P(\theta \mid D, m)=\frac{P(\theta \mid m) P(D \mid \theta, m)}{P(D \mid m)}
$$

where $\theta$ denotes the set of fitting parameters, $D$ is the data, and $m$ is the model. The vertical bar indicates that these are conditional probabilities. Each term in Bayes theorem has relevant meaning. $P(\theta m)$ is called the prior and is the probability assigned to the set of parameters before data is collected, consistent with the model. The likelihood, $P(D \mid \theta, m)$, is the probability of obtaining the data set for assumed values of the parameters and the model. Multiplication by the likelihood updates the prior in the light of the data to give the posterior, $P(\theta D, m)$. The posterior is the probability of obtaining a particular set of parameter values given the data (and the model). In general it is a multidimensional probability distribution over the space of fitting parameters. The evidence, $P(D \mid m)$, normalizes the product of the prior and likelihood. It is the probability of obtaining the data set $D$ given the model chosen. The evidence is crucial for model selection. It offers a systematic way of deciding what model best describes the data.

The posterior distribution and evidence have been calculated in a variety of ways. Techniques such as Bayesian maximum entropy and Markov Chain Monte Carlo (MCMC) path sampling methods have been used [39-41]. MCMC methods have traditionally focused on sampling the inference distribution, and often minimize the importance of the evidence [42]. These algorithms have faced sampling efficiency problems for multimodal inference distributions $[43,44]$. Recent nested sampling approaches have focused on calculation of the Bayesian evidence $[42,43]$. The prior is sampled randomly within "nested" contours of increasing likelihood [42]. In particular, the MultiNest algorithm calculates the evidence, identifies modes in multimodal posteriors, and generates random samples of the posterior distribution [44]. Modes are values of parameters where there is a local maximum in the posterior distribution. The samples can be used to calculate parameter averages, averages of predicted distributions, and derived data. We used MultiNest to determine the average apparent FRET efficiency distribution, as described shortly. For a complete explanation of the MultiNest algorithm, we refer readers to the original manuscripts [43,44]. 
We determined the FRET efficiency distribution of simulated data and of dye labeled calmodulin using a Bayesian inference approach. Photon distribution analysis (PDA) theory was used as the basic framework $[25,38,45]$. The arrival times of photons in two channels were noted. The photon counts were binned into equal, non-overlapping $300 \mu \mathrm{s} \mathrm{time} \mathrm{bins.}$ Bins containing a total of ten to sixty detector counts were used to create a two-dimensional signal histogram for the experimental data. Bins containing one to fifty-five counts were used for simulated data. The histogram contains the number of times a bin contained $S 1$ and $S 2$ detector counts in channels 1 and 2, $N(S 1, S 2)$. PDA describes how to transform the apparent FRET efficiency distribution $P\left(E_{\text {app }}\right)$ into the signal distribution $P(S 1, S 2)$ [38], according to the formula

$$
P(S 1, S 2)=\int \sum_{F 1+B 1=S 1 ; F 2+B 2=S 2} P\left(F 1 \mid F, E_{a p p}\right) P_{\langle B 1\rangle}(B 1) P_{\langle B 2\rangle}(B 2) P(S) P\left(E_{a p p}\right) d E_{a p p}
$$

where $F 1$ and $F 2$ are the number of emitted photons detected, $F=F 1+F 2, E_{a p p}$ is the apparent FRET efficiency, $B 1$ and $B 2$ are the number of background counts detected, $P(F 1 \mid$ $\left.F, E_{a p p}\right)$ is a binomial distribution with probability of success $1-E_{a p p}, P_{\langle B I\rangle}$ and $P_{\langle B 2\rangle}$ are Poisson distributions with averages $\langle B I\rangle$ and $\langle B 2\rangle$, and $P(S)$ is the probability of detecting a total number of counts $S=S 1+S 2$ in a bin. The distribution $P(S)$ (i.e. including background counts) is an approximation to the fluorescence photon distribution $P(F)$ as described by Antonik et al.[38] The signal histogram, $N(S 1, S 2)$, is a measure of the signal distribution, $P(S 1, S 2)$. Here, $N(S 1, S 2)$ and $P(S 1, S 2)$ differ by a normalization factor of the total number of counts in the signal histogram.

$P\left(E_{\text {app }}\right)$ was digitized into a grid of 100 equally spaced points. For MultiNest fits, the probability placed in each grid point was determined from a sum of Gaussian distributions. Each Gaussian was parameterized by an amplitude, $a$, width, $\sigma$, and center, $x$,

$$
P\left(E_{a p p}^{i}\right)=\Delta E_{a p p} \sum_{k} \frac{a_{k}}{\sqrt{2 \pi \sigma_{k}^{2}}} e^{\frac{-\left(E_{a p p}^{i}-x_{k}\right)^{2}}{2 \sigma_{k}{ }^{2}}}
$$

where $\Delta E_{\text {app }}$ is the grid bin width and $P\left(E_{\text {app }}{ }^{i}\right)$ is the probability at grid point $i$. The number of Gaussians, $k$, was changed in subsequent fits, and the evidence was used to determine the best model choice.

Random samples from the posterior distribution produced by MultiNest were used to calculate the average $P\left(E_{\text {app }}\right)$. Since the average $P\left(E_{\text {app }}\right)$ distribution is calculated from samples, peaks in the resulting $P\left(E_{a p p}\right)$ need not be Gaussian. The fitting model we used represents the distribution as a sum of Gaussians. After fitting based on a dataset, $P\left(E_{\text {app }}\right)$ is transformed into the FRET efficiency distribution, $P(E)$. The FRET efficiency is related to the apparent FRET efficiency by [38]

$$
E_{a p p}(E)=1-\frac{1}{1+\frac{g_{2} \varphi_{2}}{g_{1} \varphi_{1}}\left(\frac{E}{1-E}\right)+\frac{g_{2}}{g_{1}} c}
$$

where $g_{1}$ and $g_{2}$ are the detection efficiencies for the donor and acceptor data acquisition channels, $\varphi_{1}$ and $\varphi_{2}$ are the quantum yields of the donor and acceptor dyes, and $c$ is the cross talk of the donor emission into the acceptor data acquisition channel. The detection efficiencies and cross talk were measured as in Ref. [22]. 
Prior

Each parameter must be assigned a prior distribution. In the context of a Gaussian model in which each peak represents a population of protein conformations, we chose to constrain the range of $x$ to the interval $[0,1]$ (inclusive). The amplitude parameter $a_{k}$ must be constrained to the range $[0,1]$ because the entire distribution is constructed from the sum of a finite number of Gaussians. The width of the distribution must have a lower bound of zero, as a negative width has no meaning. The upper bound on the width parameter could in principle stretch to infinity. As the width increases the peaked Gaussian distribution flattens into a line, however. Since $P\left(E_{\text {app }}\right)$ is expected to contain peaks, we chose to constrain the width of the distribution to the range $[0,1]$. We choose the prior probability distribution for each parameter to be flat within the chosen bounds (a top hat distribution).

\section{Likelihood}

A Poisson distribution was chosen for the likelihood

$$
\ln P(D \mid \theta, m)=\sum_{i} D_{i} \ln M_{i}-M_{i}-\ln D_{i} !
$$

where $D_{i}$ is a data point in the signal histogram $N(S 1, S 2), M_{i}$ is a mock data point calculated from the model for a specific value of the parameters, and the sum is taken over all data points in the signal histogram indexed by $i$.

\section{Classic Maximum Entropy}

For comparison, the classic maximum entropy method was used to recover the joint distribution for the apparent FRET efficiency and the fluorescence photons, $P\left(E_{a p p}, F\right)$, modeled as a discretized joint distribution [22]. In addition, a hidden distribution consisting of multiple layers of $P\left(E_{a p p}, F\right)$ was used to introduce correlations between adjacent grid points in each layer $[22,41,46]$. The recovered joint distribution was then marginalized over the fluorescence photon dimension to give $P\left(E_{\text {app }}\right)$. The result was compared with the MultiNest sampling approach. A description of cMEM for sm-FRET burst data has been published previously [22].

\section{Results}

The MultiNest model was tested with simulated data. Figure 3 shows the fitting results. The left column compares the simulated $P(E)$ distribution (black) to the fit (red). Fits were undertaken with varying numbers of Gaussians in the model. The fit from the model with the largest Bayesian evidence is plotted in the left column. The right hand column shows the $\log$ Bayesian evidence for each fit. The fitted $P(E)$ distributions corresponding to that model with the largest evidence accurately match the simulated distributions.

The simulated data were also matched against cMEM. The number of layers $(n L)$ in the hidden distribution was varied from 0 to 6 in successive fits. The cMEM model with the largest $\log$ evidence for each simulation is tabulated in Table 1. A five layer cMEM model was optimal for each simulated data set. The MultiNest model with the largest evidence for each data set is displayed in Table 1 for comparison. In all fits of simulated data, the evidence was larger for the MultiNest models than the cMEM models, showing that MultiNest succeeds in modeling the simulated data better than cMEM.

CaM-44-117-AF488-AF594 and CaM-44-117-AF488-TR bursts were measured in both high $\mathrm{Ca}^{2+}$ and low $\mathrm{Ca}^{2+}$ buffers. Figure 4 shows the FRET efficiency distribution determined by fitting the signal histogram using MultiNest and cMEM fitting methods. All 
distributions show a donor-only peak with an apparent FRET efficiency near zero. The fit distributions determined by MEM and MultiNest are similar in all panels. Panel A reveals two FRET states for CaM-44-117-AF488-AF594 in high $\mathrm{Ca}^{2+}$. In low $\mathrm{Ca}^{2+}$, CaM-44-117AF488-AF594 shows a single FRET state which exhibits a small shoulder at lower FRET efficiency, in Panel B. Panel C shows distributions of CaM-44-117-AF488-TR in high $\mathrm{Ca}^{2+}$. The greatest difference in the FRET efficiency distributions determined by the two techniques is found in panel C. Maximum entropy recovers three distinct FRET states from the data. MultiNest, in contrast, recovers two FRET states, but with a shoulder.

CaM-44-117-AF488-TR in low $\mathrm{Ca}^{2+}$ is displayed in panel $\mathrm{D}$.

The FRET efficiency distributions of calmodulin in an excess of CaMKII peptide are displayed in Figure 5. Again, all fits display a donor-only peak at an apparent FRET efficiency of zero. In high $\mathrm{Ca}^{2+}$, CaM-44-117-AF488-AF594 with CaMKII peptide displays a single FRET state centered at 0.79; see Fig 5A. CaM-44-117-AF488-AF594 with CaMKII peptide in low $\mathrm{Ca}^{2+}$ has a large FRET state centered at 0.77 , with a shoulder to the left. In high $\mathrm{Ca}^{2+}$, CaM-44-117-AF488-TR has a larger peak centered at 0.80, and a small amount of probability flanking each side of the peak; see Fig 5C. CaM-44-117-AF488-TR in low $\mathrm{Ca}^{2+}$ with CaMKII peptide is centered at 0.91 with a shoulder to the left of the peak.

The optimal number of Gaussians in the MultiNest model for the experimental data was determined from the Bayesian evidence. Figure 6 shows typical plots of the log evidence versus number of Gaussians (for MultiNest fits), or versus the number of blurring layers (for cMEM). Figure 6A plots the log evidence for fits of CaM-44-117-AF488-AF594 in high $\mathrm{Ca}^{2+}$ for MultiNest. Figure 6B plots the log evidence for fits of CaM-44-117-AF488-AF594 in high $\mathrm{Ca}^{2+}$ with differing numbers of blurring layers. In both panels the evidence rises to a maximum and then begins to decrease as more layers or Gaussians are added to the models. Similar behavior was observed for all data sets analyzed.

For each data set, the cMEM and MultiNest models that produced the highest evidence were plotted. Table 2 shows the log evidence for the best model in the MEM and MultiNest framework. With the exception of CaM-44-117-AF488-TR in low $\mathrm{Ca}^{2+}$ with CaMKII peptide data, the cMEM models produced larger evidences than the MultiNest models, indicating that cMEM represents a better model, given this data.

An example of cMEM recovery is shown in Figure 7. The data correspond to CaM-44-117AF488-AF594 with and without CaMKII peptide in high $\mathrm{Ca}^{2+}$ buffer. Figures 7A and 7B show $P\left(E_{\text {app }}, F\right)$. The joint distribution can be marginalized to obtain the apparent FRET efficiency distribution $P\left(E_{\text {app }}\right)$ or the fluorescence photon distribution $P(F)$. Figures $7 \mathrm{C}$ and 7D show $P(F)$ calculated from panels A and B. $P(F)$ is also compared to the signal distribution $P(S)$ calculated from the data sets. Once $P\left(E_{\text {app }}\right)$ is obtained by marginalization, the distribution can be transformed into $P(E)$. Figures $4 \mathrm{~A}$ and $5 \mathrm{~A}$ show $P(E)$ for these datasets.

The quality of the distributions recovered by MultiNest and cMEM can be compared by computation of the fitting residuals. An example is shown in Figure 8. Figure 8A shows the data for CaM-44-117-AF488-AF594 with CaMKII peptide in high $\mathrm{Ca}^{2+}$. The data is shown as a two dimensional signal histogram, $N(S 1, S 2)$. The average $P\left(E_{a p p}\right)$ distribution from MultiNest was used to generate expected ( mock) data. The mock data were used in conjunction with the experimental data to calculate the residuals. The residuals calculated for the mock data set and actual data set are shown in Figure 8B. The residuals for mock data generated from the cMEM are shown in Figure 8C. The MultiNest model used three Gaussians, and the cMEM model contained four layers of blurring. 


\section{Discussion}

\section{Classic maximum entropy and MultiNest}

The MultiNest model produced larger evidences than cMEM for each simulated data set. For experimental data, however, cMEM produced larger evidence values than MultiNest, with the exception of low $\mathrm{Ca}^{2+} \mathrm{CaM}-44-117-\mathrm{AF} 488-\mathrm{TR}$ with CaMKII peptide. This observation shows that the cMEM model was more appropriate for the experimental data sets, whereas the MultiNest model was more appropriate for the simulated data sets, suggesting that MultiNest models are particularly well suited to situations in which Gaussian distributions can be expected.

There are several differences between the cMEM and MultiNest models. The number of parameters in each model is very different. The Gaussian model in MultiNest contains three parameters for each Gaussian. cMEM was not constrained to any parameterized function. Each point in the discretized $P\left(E_{a p p}, F\right)$ distribution was a variable parameter. For the calmodulin fits, there were 100 grid points in the $E_{\text {app }}$ dimension and 51 grid points in the $F$ dimension for a minimum number of variable parameters in the cMEM model of 5100. This corresponds to a cMEM model containing only one layer. However, multilayered models consistently gave a higher evidence value than a one-layer non-blurred model. Each layer added to the model was blurred by a Gaussian footprint function by a factor of 2 greater than the previous layer. This reduces the effective degrees of freedom for each layer by adding correlations between adjacent grid points [41].

Built into the Bayesian evidence is a dependence on the number of parameters. For two fits that reproduce the data well but have differing numbers of parameters, the fit with fewer parameters will have larger evidence [47]. This parameter dependence would work in favor of the MultiNest model used in this paper. However, cMEM models with multiple layers of blurring fit the experimental data better in most cases examined here (though not all), increasing the evidence above that of MultiNest. Although the residuals are not explicitly minimized in the Bayesian framework, the likelihood penalizes large differences between the experimental data and model-generated mock data. The residuals from Figure $8 \mathrm{~B}$ and $8 \mathrm{C}$ show systematic deviations and larger amplitudes for the MultiNest model than cMEM.

Another difference between models was the approximation of setting $P(F)$ equal to $P(S)$ in the PDA (equation 2) for the MultiNest model. The approximation is not needed in the cMEM model, as $P\left(E_{a p p}, F\right)$ was fitted directly. Figures 7A and 7B show the two dimensional $P\left(E_{\text {app }}, F\right)$ distribution recovered by cMEM for CaM-44-117-AF488-AF594, with and without CaMKII peptide in high $\mathrm{Ca}^{2+}$. The $P(F)$ distribution is found by marginalizing over all allowed values of $E_{a p p} . P(S)$ is compared to $P(F)$ in Figures 7C and 7D. In both cases $P(F)$ closely resembles $P(S)$. However, cMEM has the freedom to utilize different shapes for $P(F)$ at each FRET efficiency - the fit is two-dimensional, whereas the signal approximation in the MultiNest model must use the same shape, $P(S)$, across all FRET efficiencies, assuming that $F$ and $E$ are independent. This assumption does not hold if the quantum yields of the donor and acceptor dye are different. Figure 9 shows $P(F)$ calculated over ranges of $E_{\text {app }}$ associated with the donor only and CaMKII peptide bound CaM-44-117-AF488-AF594 in high $\mathrm{Ca}^{2+}$. Fewer photons are likely to be detected by bursts with a FRET efficiency around 0.84 than for the donor only species. This is consistent with the fact that the quantum yield of AF594 is lower than that of AF488, and energy transfer from AF488 to AF594 will result in a species that is not as bright as a species with no energy transfer. Kalinin et al. have shown that utilizing a variable fluorescence distribution improves the fit $[25,45]$. This may contribute to the improved fit for cMEM with regard to the experimental data. In future work, the MultiNest model could be adjusted to include an analytical form for the $P(F)$ decay. This would require more model parameters, but would 
allow the joint distribution $P\left(E_{a p p}, F\right)$ to be modeled. Again, the evidence could be used to determine whether the new model is better suited to analyze the data.

The simulated data was ideal for use with the signal approximation and MultiNest model. Its purpose was to test our algorithms. In MultiNest, the $P(E)$ distribution is modeled as a sum of Gaussians. The simulated data was constructed with this assumption in mind. Two Gaussian $P(E)$ states were used, each having the same $P(F)$ shape. The centers of the two Gaussian distributions used to simulate $P(E)$ were set closer in successive simulations until the two peaks were no longer discernible in the $P(E)$ distribution; see Figures 3 A,C,E,G. The difference in log evidence between a two-Gaussian or single Gaussian fit was 5460.7, 182.8, 12.6, and -1.3 for simulations 1, 2, 3 and 4 respectively (Figures 3 B, D, F, G). As the centers of the underlying Gaussians were moved closer together in the first three simulations, the fit to a single Gaussian improved, but was still not as good as the twoGaussian model. In the fourth simulation, the single-Gaussian model was adequate to specify the $P\left(E_{a p p}\right)$ distribution, with a slightly larger evidence. Models with three and four Gaussians had lower evidence than the two Gaussian model for all simulated datasets. This demonstrates the power of the Bayesian evidence for model selection. Overall, the MultiNest model performed as expected, fitting the simulated data well with few parameters and with larger evidence than cMEM. Furthermore, the residuals from the MultiNest fits with largest evidences were not biased.

\section{Dye interactions and peptide binding}

The FRET distributions of CaM-44-117-AF488-AF594 and CaM-44-117-AF488-TR in the absence of CaMKII peptide are distinct. The Förster distance, $R_{0}$, for AF488-AF594 on calmodulin was estimated as $56 \AA$, and for AF488-TR the distance is $54 \AA$. The dye linker lengths differ by three carbon to carbon sp3 hybridized bonds. Both dye pairs are zwitterionic. However, Texas Red carries charge that is overall neutral, whereas AF594 has an overall charge of -1 . Dyes with similar Förster distances and linker lengths that do not interact with each other or the protein are expected to produce similar FRET distributions. The difference in distributions determined for these dye pairs in the absence of CaMKII suggests more severe differences, which cannot be accounted for merely by differences in $R_{O}$ or linker length. It appears that there is a difference in protein-dye interactions.

In the presence of the CaMKII binding domain, both $\mathrm{Ca}^{2+}$ bound CaM-44-117-AF488AF594 and CaM-44-117-AF488-TR give largely the same distribution. The dissociation constant for $\mathrm{Ca}^{2+}$ calmodulin and CaMKII is $100 \pm 35 \mathrm{pM}[48,49]$. Thus, at the concentrations used in our measurements at high $\mathrm{Ca}^{2+}$, nearly all calmodulin was bound to the CaMKII peptide. CaM binds to the CaMKII peptide in a classic CaM binding motif, in which hydrophobic regions exposed in $\mathrm{Ca}^{2+}$-activated $\mathrm{CaM}$ interact with hydrophobic residues of the CaMKII binding domain to form the compact structure shown in Figure 2B [50]. Figure 4 shows that the FRET distributions differ for CaM-44-117-AF488-TR and CaM-44-117-AF488-AF594 in the absence of CaMKII peptide. The convergence of these distributions to nearly identical distributions with a main FRET peak centered at a FRET efficiency of 0.80 for both CaM-44-117-AF488-TR and CaM-44-117-AF488-AF594 in the presence of peptide suggests that interactions (or noninteractions) of the dye pairs with the protein are similar in the peptide-bound conformation. Dyes could interact with the protein at a number of surface sites including, for calmodulin at high $\mathrm{Ca}^{2+}$, the hydrophobic domains involved in target binding. The FRET distributions for CaM-44-117-AF488-TR and CaM-44-117-AF488-AF594 in the presence of CaMKII peptide are not quite identical. A small shoulder appears with the TR acceptor around $\mathrm{E}=0.45$, suggesting the presence of a minor population of CaM-44-117-AF488-TR for which TR interacts differently with the protein. In low $\mathrm{Ca}^{2+}$ conditions, in which the hydrophobic binding domains of $\mathrm{CaM}$ are buried [51], the distributions for CaM-44-117 with both dye pairs in the presence and 
absence of CaMKII peptide are similar, consistent with the notion that the dye pairs interact differently with the hydrophobic domains accessible under high- $\mathrm{Ca}^{2+}$ conditions in the absence of target peptide.

\section{Conclusion}

The FRET distributions of CaM-44-117-AF488-AF594 and CaM-44-117-AF488-TR imply that it is vital to understand the interactions between dyes and protein for interpretation of calmodulin conformations from sm-FRET. Experiments with multiple dye pairs are essential to elucidate differences in FRET states due to the nature of the dye. The multiple FRET states observed with Texas Red at high $\mathrm{Ca}^{2+}$ suggest that TR interacts differently with the high- $\mathrm{Ca}^{2+}$ protein than AF594.

The MultiNest Bayesian analysis generates satisfactory models for the underlying FRET distribution from sm-FRET burst data. In our implementation, MultiNest uses Gaussian models for the FRET distributions, so it is not surprising that MultiNest is particularly successful in fitting data for which Gaussian distributions are expected. For the experimental FRET distributions we found that cMEM provided a better model than MultiNest, judged by the Bayesian evidence. MultiNest is adaptable to different models, and the calculation of the Bayesian evidence allows model selection. We believe that MultiNest will be particularly appropriate for testing specific models for the FRET distribution (Gaussian or some other form). The model we used with MultiNest could also be modified to incorporate a specific model for the fluorescence distribution $P(F)$. This makes MultiNest a viable choice for parameterized model selection in single molecule spectroscopy.

\section{Acknowledgments}

We thank the National Science Foundation (CHE-0710515) for financial support. We thank the Protein Production Group at the University of Kansas COBRE Center in Protein Structure and Function (NIH RR-017708) for production of CaM-44-117. MSD is grateful for support provided by the NIH Dynamic Aspects of Chemical Biology Training Grant (GM08545).

\section{References}

1. Ha T, Enderle T, Ogletree DF, Chemla DS, Selvin PR, Weiss S. Proc Natl Acad Sci USA. 1996; 93:6264. [PubMed: 8692803]

2. Talaga DS, Lau WL, Roder H, Tang J, Jia Y, DeGrado WF, Hochstrasser RM. Proc Natl Acad Sci USA. 2000; 97:13021. [PubMed: 11087856]

3. Schuler B, Lipman EA, Eaton WA. Nature. 2002; 419:743. [PubMed: 12384704]

4. Margittai M, Widengren J, Schweinberger E, Schroder GF, Felekyan S, Haustein E, Konig M, Fasshauer D, Grubmuller H, Jahn R, Seidel CAM. Proc Natl Acad Sci USA. 2003; 100:15516. [PubMed: 14668446]

5. Dale RE, Eisinger J, Blumberg WE. Biophys J. 1979; 26:161. [PubMed: 262414]

6. Eggeling C, Fries JR, Brand L, Gunther R, Seidel CAM. Proc Natl Acad Sci USA. 1998; 95:1556. [PubMed: 9465054]

7. Sisamakis E, Valeri A, Kalinin S, Rothwell PJ, Seidel CAM. Meth Enzymol. 2010; 475:455. [PubMed: 20627168]

8. Dahan M, Deniz AA, Ha T, Chemla DS, Schultz PG, Weiss S. Chem Phys. 1999; 247:85.

9. Kalinin S, Sisamakis E, Magennis SW, Felekyan S, Seidel CAM. J Phys Chem B. 2010; 114:6197. [PubMed: 20397670]

10. Kapanidis AN, Lee NK, Laurence TA, Doose S, Margeat E, Weiss S. Proc Natl Acad Sci USA. 2004; 101:8936. [PubMed: 15175430]

11. Kapanidis AN, Laurence TA, Lee NK, Margeat E, Kong XX, Weiss S. Acc Chem Res. 2005; 38:523. [PubMed: 16028886] 
12. Eggeling C, Berger S, Brand L, Fries JR, Schaffer J, Volkmer A, Seidel CAM. J Biotech. 2001; 86:163.

13. Muller BK, Zaychikov E, Brauchle C, Lamb DC. Biophys J. 2005; 89:3508. [PubMed: 16113120]

14. Witkoskie JB, Cao J. J Chem Phys. 2004; 121:6373. [PubMed: 15446934]

15. Kou SC, Sunney Xie X, Liu JS. J R Stat Soc: Ser C. 2005; 54:469.

16. Witkoskie JB, Cao J. J Phys Chem B. 2008; 112:5988. [PubMed: 18266353]

17. Bronson JE, Fei JY, Hofman JM, Gonzalez RL, Wiggins CH. Biophys J. 2009; 97:3196. [PubMed: 20006957]

18. Gopich IV, Szabo A. J Phys Chem B. 2009; 113:10965. [PubMed: 19588948]

19. Taylor JN, Makarov DE, Landes CF. Biophys J. 2010; 98:164. [PubMed: 20074517]

20. Ensign DL, Pande VS. J Phys Chem B. 2010; 114:280. [PubMed: 20000829]

21. Backovic M, Price ES, Johnson CK, Ralston JP. J Chem Phys. 2011; 134:15.

22. DeVore MS, Gull SF, Johnson CK. J Phys Chem B. 2012; 116:4006. [PubMed: 22338694]

23. Watkins LP, Chang HY, Yang H. J Phys Chem A. 2006; 110:5191. [PubMed: 16610843]

24. Hanson JA, Duderstadt K, Watkins LP, Bhattacharyya S, Brokaw J, Chu JW, Yang H. Proc Natl Acad Sci USA. 2007; 104:18055. [PubMed: 17989222]

25. Kalinin S, Felekyan S, Antonik M, Seidel CAM. J Phys Chem B. 2007; 111:10253. [PubMed: 17676789]

26. Chazin WJ. Acc Chem Res. 2011; 44:171. [PubMed: 21314091]

27. Babu YS, Sack JS, Greenhough TJ, Bugg CE, Means AR, Cook WJ. Nature. 1985; 315:37. [PubMed: 3990807]

28. Wilson MA, Brunger AT. J Mol Biol. 2000; 301:1237. [PubMed: 10966818]

29. Chattopadhyaya R, Meador WE, Means AR, Quiocho FA. J Mol Biol. 1992; 228:1177. [PubMed: 1474585]

30. Fallon JL, Quiocho FA. Structure. 2003; 11:1303. [PubMed: 14527397]

31. Meador WE, Means AR, Quiocho FA. Science. 1993; 262:1718. [PubMed: 8259515]

32. Anderson ME. Pharmacol Ther. 2005; 106:39. [PubMed: 15781121]

33. Braun AP, Schulman H. Annu Rev Physiol. 1995; 57:417. [PubMed: 7778873]

34. Tobimatsu T, Fujisawa H. J Biol Chem. 1989; 264:17907. [PubMed: 2553697]

35. Meador WE, Means AR, Quiocho FA. Science. 1993; 262:1718. [PubMed: 8259515]

36. Allen MW, Urbauer RJB, Zaidi A, Williams TD, Urbauer JL, Johnson CK. Anal Biochem. 2004; 325:273. [PubMed: 14751262]

37. Price ES, DeVore MS, Johnson CK. J Phys Chem B. 2010; 114:5895. [PubMed: 20392129]

38. Antonik M, Felekyan S, Gaiduk A, Seidel CAM. J Phys Chem B. 2006; 110:6970. [PubMed: 16571010]

39. Gelman A, Meng XL. Stat Sci. 1998; 13:163.

40. Skilling, J. Maximum Entropy and Bayesian Methods. Skilling, J., editor. Kluwer Academic Publishers; Dordrecht: 1989. p. 45

41. Gull, SF. Maximum Entropy and Bayesian Methods. Skilling, J., editor. Kluwer Academic Publishers; Dordrecht: 1989. p. 53

42. Skilling J. Bayesian Anal. 2006; 1:833.

43. Feroz F, Hobson MP. Mon Not R Astron Soc. 2008; 384:449.

44. Feroz F, Hobson MP, Bridges M. Mon Not R Astron Soc. 2009; 398:1601.

45. Kalinin S, Felekyan S, Valeri A, Seidel CAM. J Phys Chem B. 2008; 112:8361. [PubMed: 18570393]

46. Gull, SF.; Skilling, J. Quantified Maximum Entropy Memsys5 Users’ Manual. Maximum Entropy Data Consultants Ltd; 1999.

47. Gull, SF. Maximum-Entropy and Bayesian Methods in Science and Engineering. Erickson, GJ.; Smith, CR., editors. Kluwer Academic Publishers; Dordrecht, Boston: 1988. p. 53

48. Allen MW, Bieber Urbauer RJ, Johnson CK. Anal Chem. 2004; 76:3630. [PubMed: 15228334]

49. Evans TI, Shea MA. Proteins. 2009; 76:47. [PubMed: 19089983] 
50. Meador WE, Means AR, Quiocho FA. Science. 1992; 257:1251. [PubMed: 1519061]

51. Zhang M, Tanaka T, Ikura M. Nat Struct Biol. 1995; 2:758. [PubMed: 7552747] 


\section{Highlights}

We analyzed single-molecule burst FRET data by Bayesian nested sampling.

MultiNest accurately recovered FRET distributions in tests on simulated data.

We measured single-molecule FRET for calmodulin labeled at sites 44 and 117.

Measured FRET distributions depended significantly on the dye pair used.

FRET distributions collapsed to one peak for calmodulin bound to CaMKII peptide. 


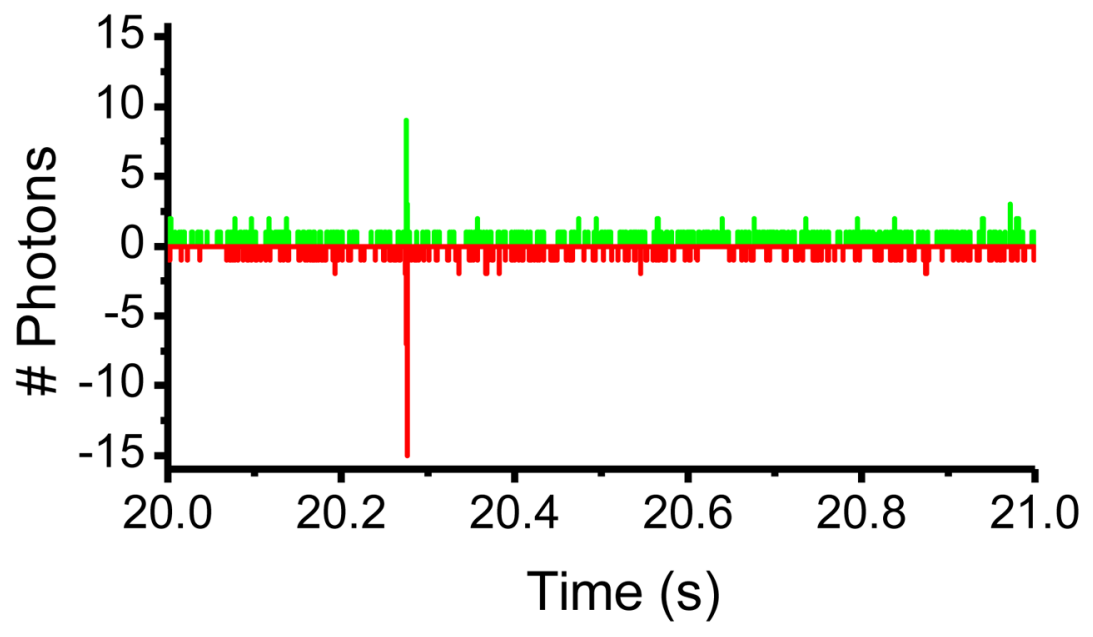

Figure 1.

Photons detected in two detection channels binned into $300 \mu \mathrm{s}$ time bins. Green corresponds to photons detected in the channel monitoring donor dye emission. Red corresponds to photons detected in the channel monitoring acceptor dye emission. The number of photons detected in the acceptor channel was multiplied by -1 for clarity in the plot. The origin of the time axis is the start of the experiment. Data are for CaM-44-117-AF488-AF594 in high $\mathrm{Ca}^{2+}$ buffer. 


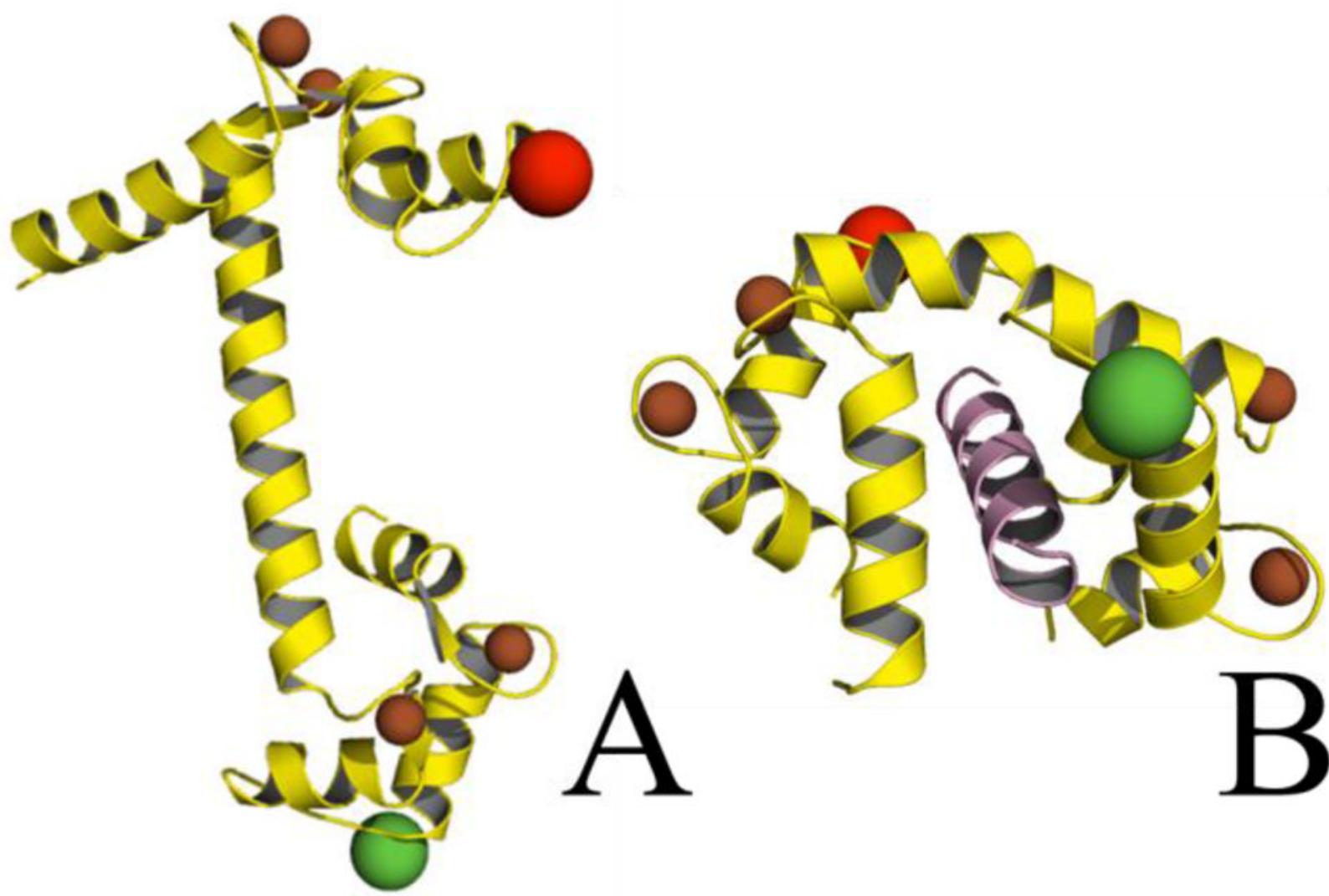

Figure 2.

A) $\mathrm{Ca}^{2+}$ calmodulin, PDB: 1CLL B) $\mathrm{Ca}^{2+}$ calmodulin bound to CaMKII peptide fragment (pink), PDB 1CDM. Both panels) $\mathrm{Ca}^{2+}$ ions are brown. Dye labeling site on residue 44 is shown as a red sphere. The dye labeling site on residue 117 is shown as a green sphere. 

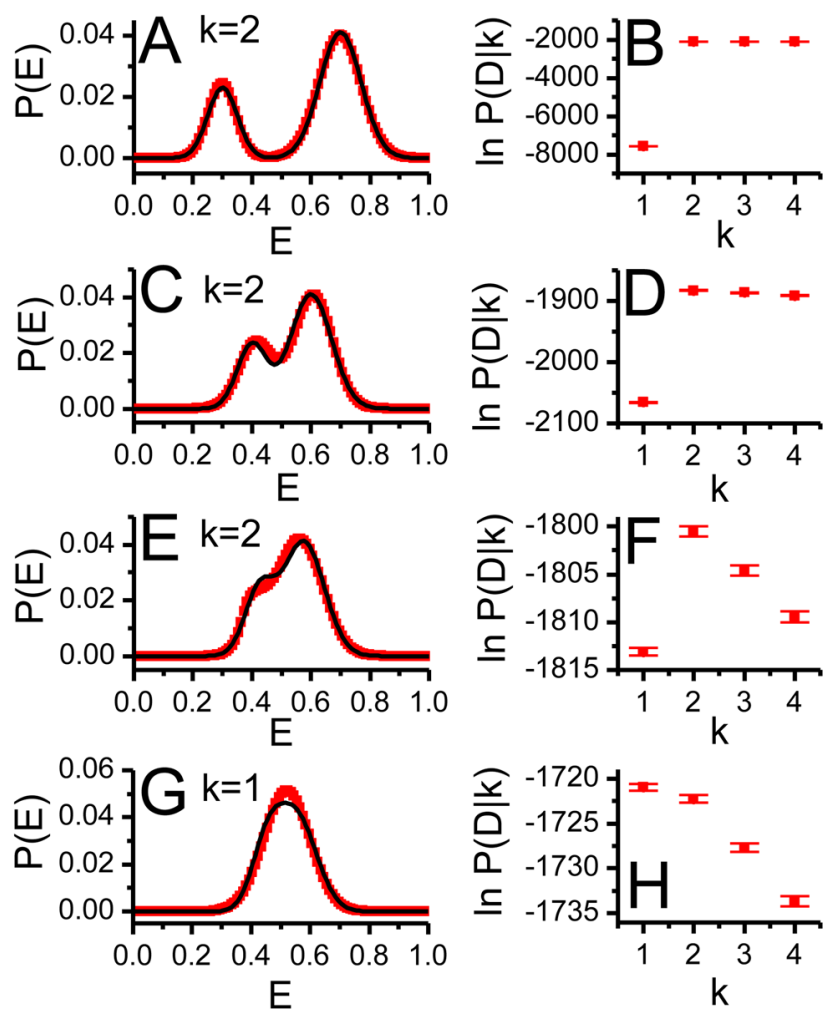

Figure 3.

Left column: Red squares show the distribution determined by the MultiNest model having highest log evidence. The black line is the simulated distribution. Right column: log evidence for fits with different numbers, $k$, of Gaussians in the MultiNest model. (A) and (B) simulation 1. (C) and (D) simulation 2. (E) and (F) simulation 3. (G) and (H) simulation 4. 

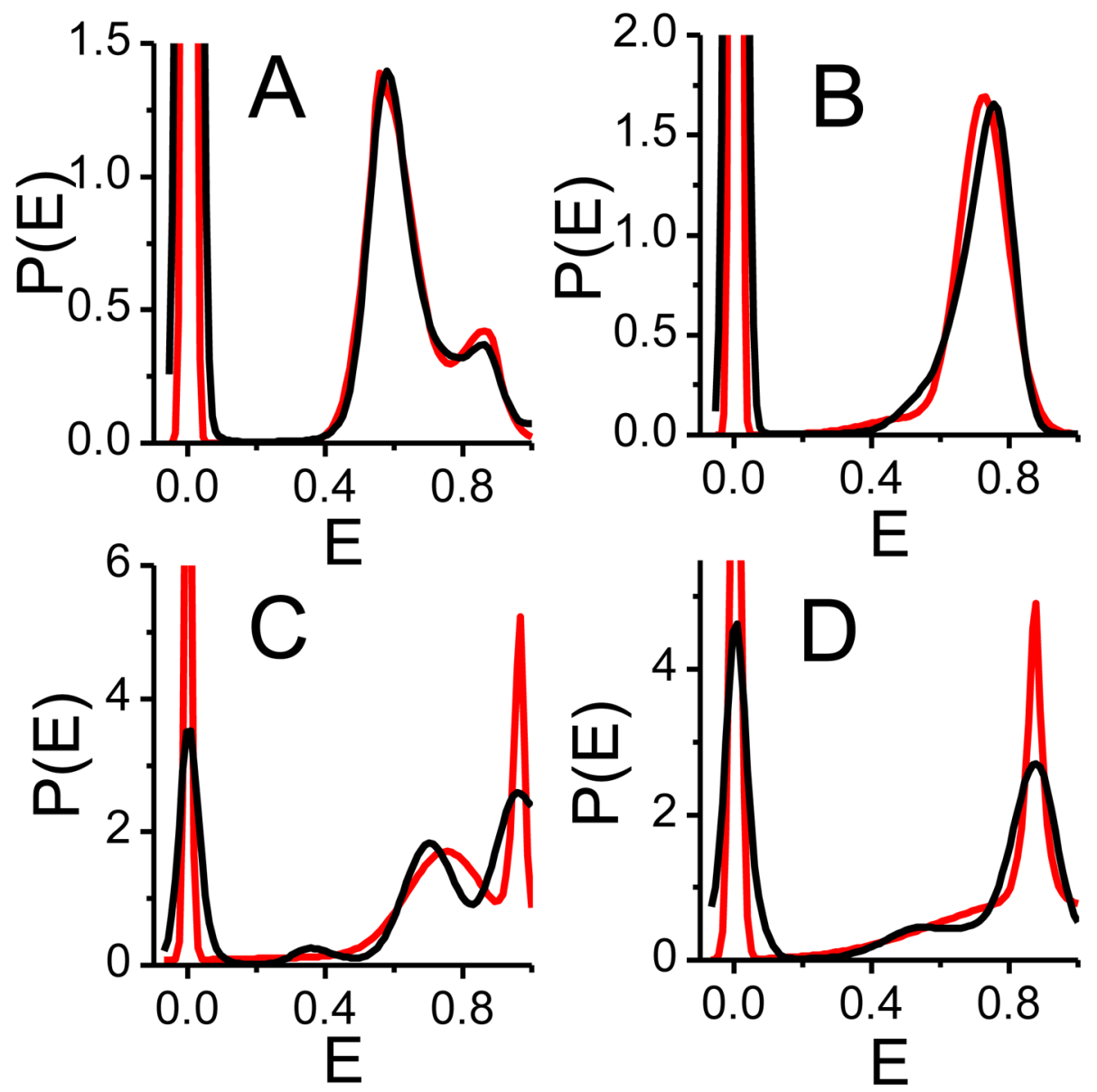

Figure 4.

FRET efficiency distributions determined by the classic maximum entropy method (black) and MultiNest (red) A) $\mathrm{Ca}^{2+}$ bound CaM-44-117-AF488-AF594 B) $\mathrm{Ca}^{2+}$ free CaM-44-117AF488-AF594 C) $\mathrm{Ca}^{2+}$ bound CaM-44-117-AF488-TR D) $\mathrm{Ca}^{2+}$ free CaM-44-117-AF488TR 

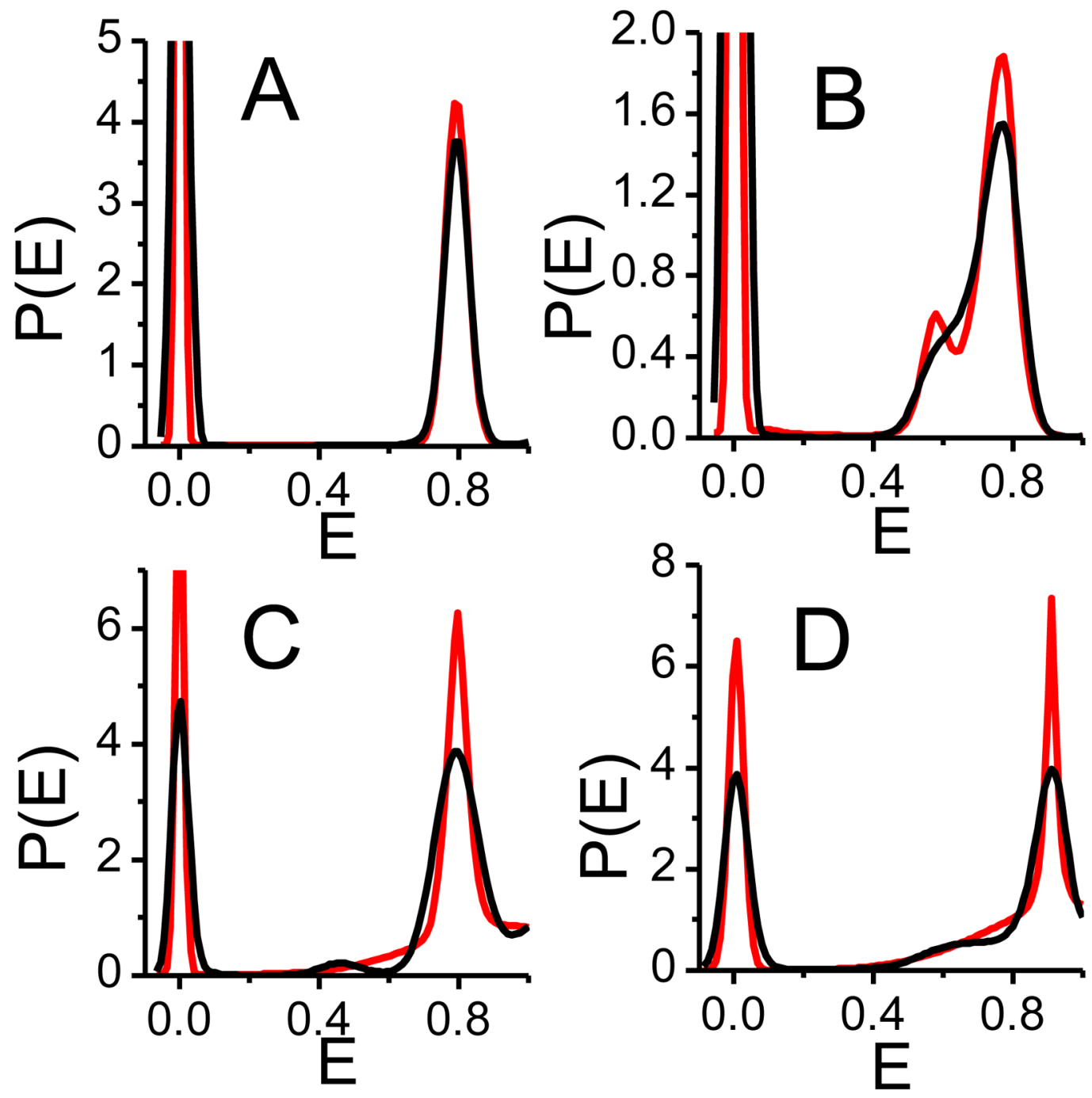

Figure 5.

FRET efficiency distributions of calmodulin in the presence of CaMKII peptide (290-309), determined by the classic maximum entropy method (black), and MultiNest (red) A) $\mathrm{Ca}^{2+}$ bound CaM-44-117-AF488-AF594 with CaMKII peptide B) $\mathrm{Ca}^{2+}$ free CaM-44-117-AF488AF594 with CaMKII peptide C) $\mathrm{Ca}^{2+}$ bound CaM-44-117-AF488-TR with CaMKII peptide D) $\mathrm{Ca}^{2+}$ free CaM-44-117-AF488-TR with CaMKII peptide. 

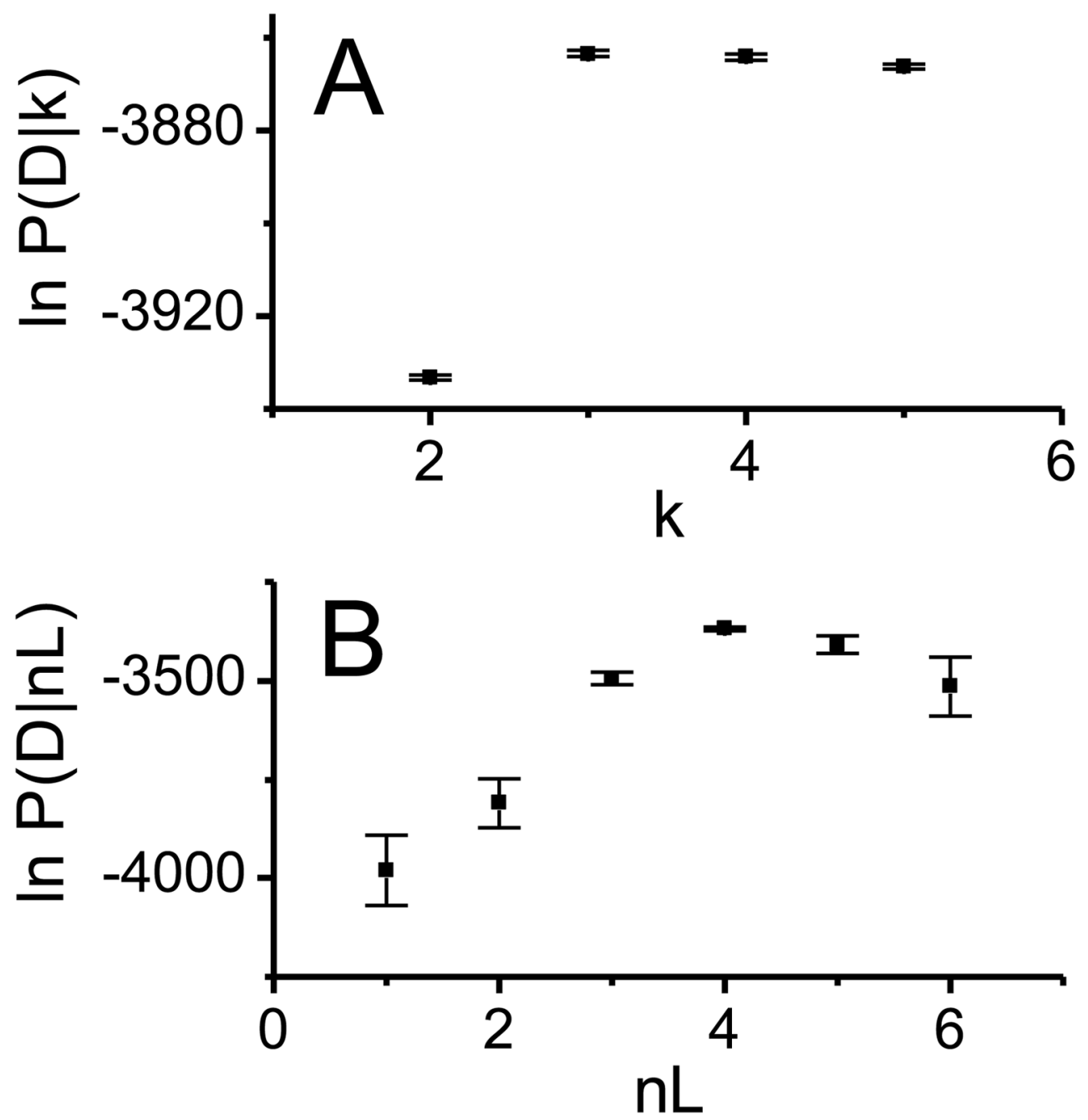

Figure 6.

Log evidence plots for $\mathrm{Ca}^{2+}$ bound CaM-44-117-AF488-AF594. A) MultiNest fits with differing numbers of Gaussian distributions in the model. B) MEM fits with multiple layers of blurring in the model. 

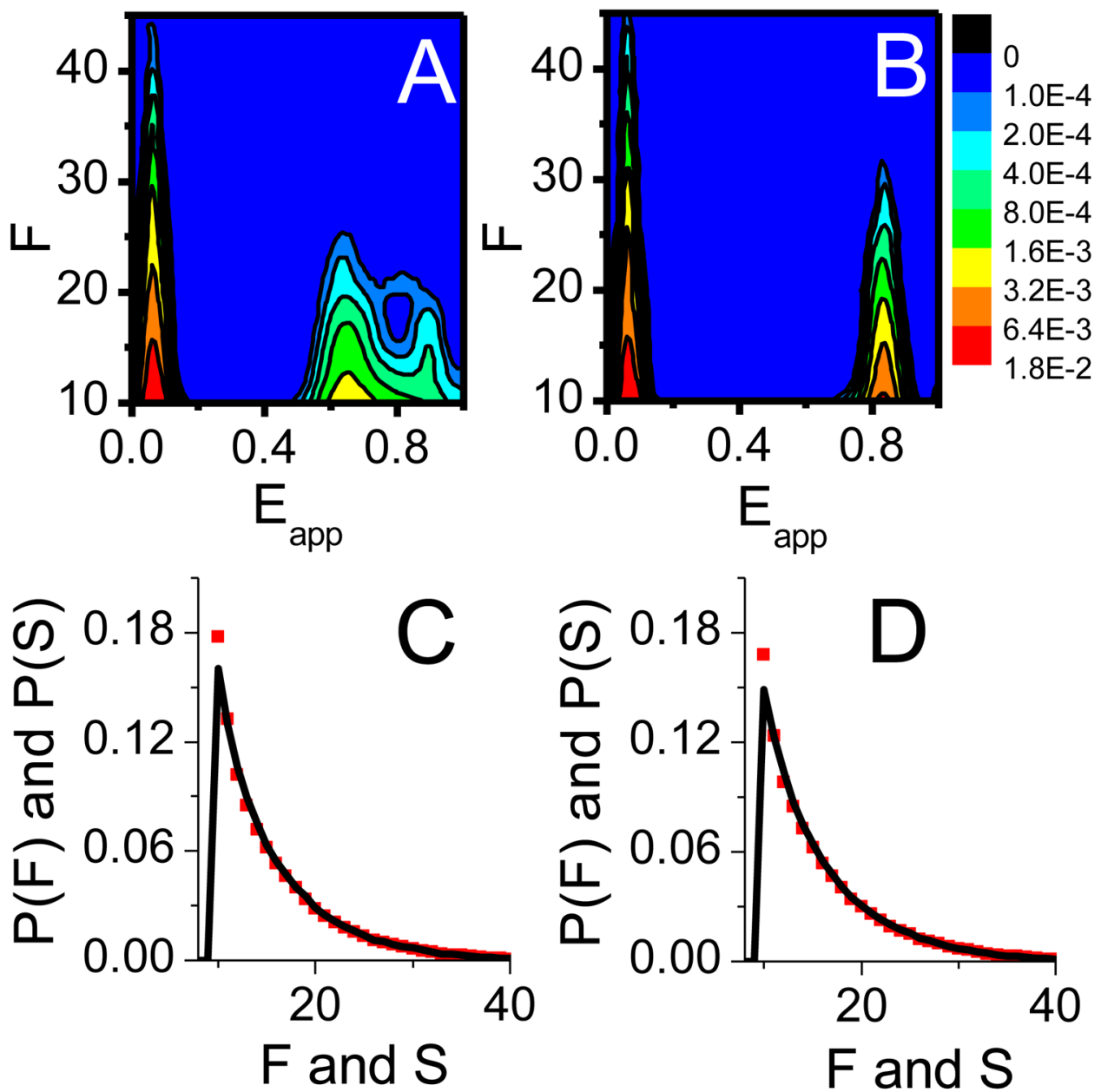

Figure 7.

A) $P\left(E_{a p p}, F\right)$ distribution recovered by cMEM for CaM-44-117-AF488-AF594 in high $\mathrm{Ca}^{2+}$. B) $P\left(E_{a p p}, F\right)$ distribution recovered by cMEM for CaM-44-117-AF488-AF594 with CaMKII peptide in high $\mathrm{Ca}^{2+}$. C) $P(F)$ distribution (red squares) and $P(S)$ signal distribution (black line) for CaM-44-117-AF488-AF594 in high $\mathrm{Ca}^{2+}$. D) $P(F)$ distribution (red squares) and $P(S)$ signal distribution (black line) for CaM-44-117-AF488-AF594 with CaMKII peptide in high $\mathrm{Ca}^{2+}$. 

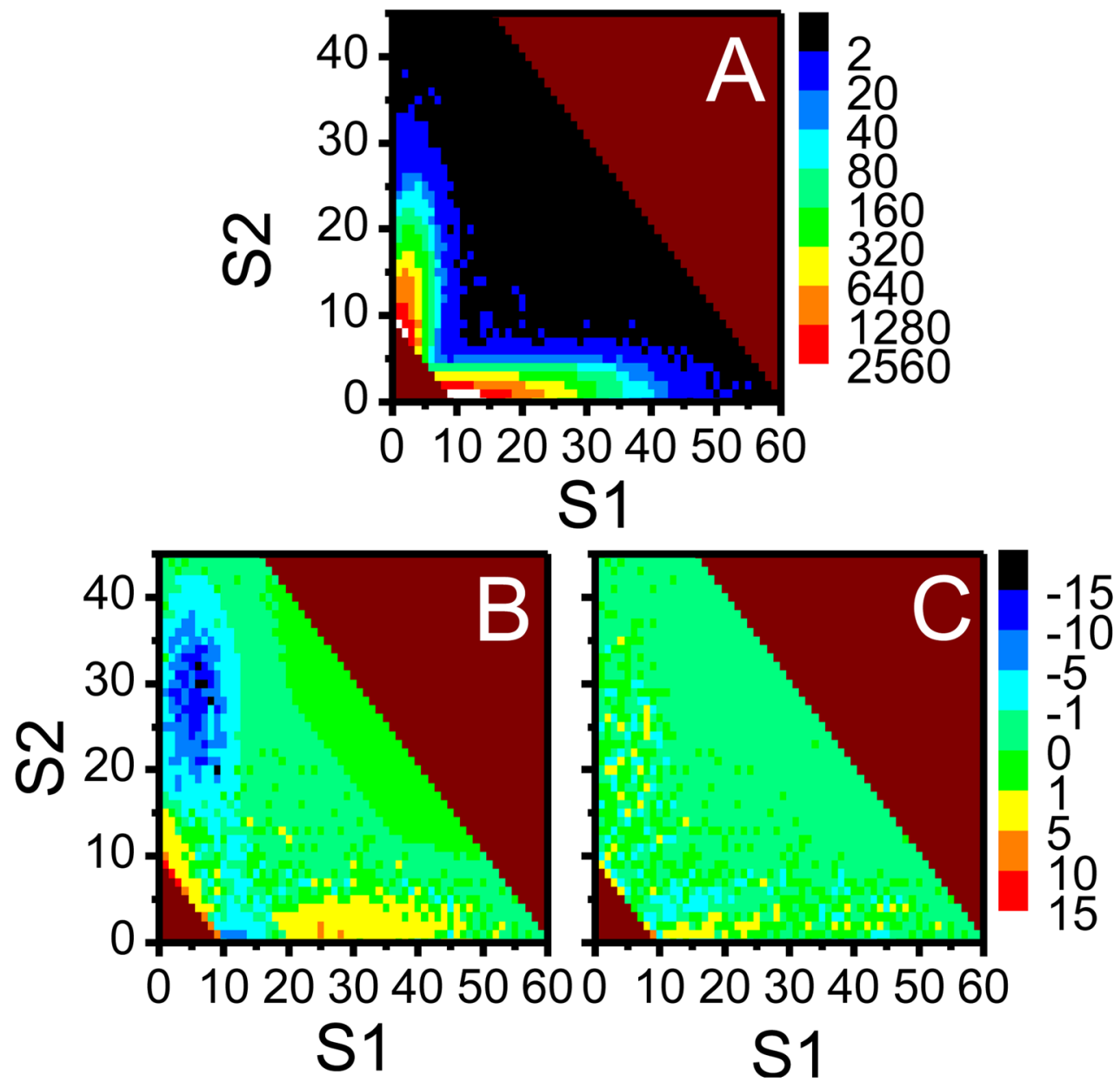

Figure 8.

CaM-44-117-AF488-AF594 with CaMKII peptide in high $\mathrm{Ca}^{2+}$ A) Two dimensional signal histogram $N(S 1, S 2)$ data. B) Fitting residuals from a three Gaussian model MultiNest fit. C) Fitting residuals from a cMEM fit with four layers of blurring. 


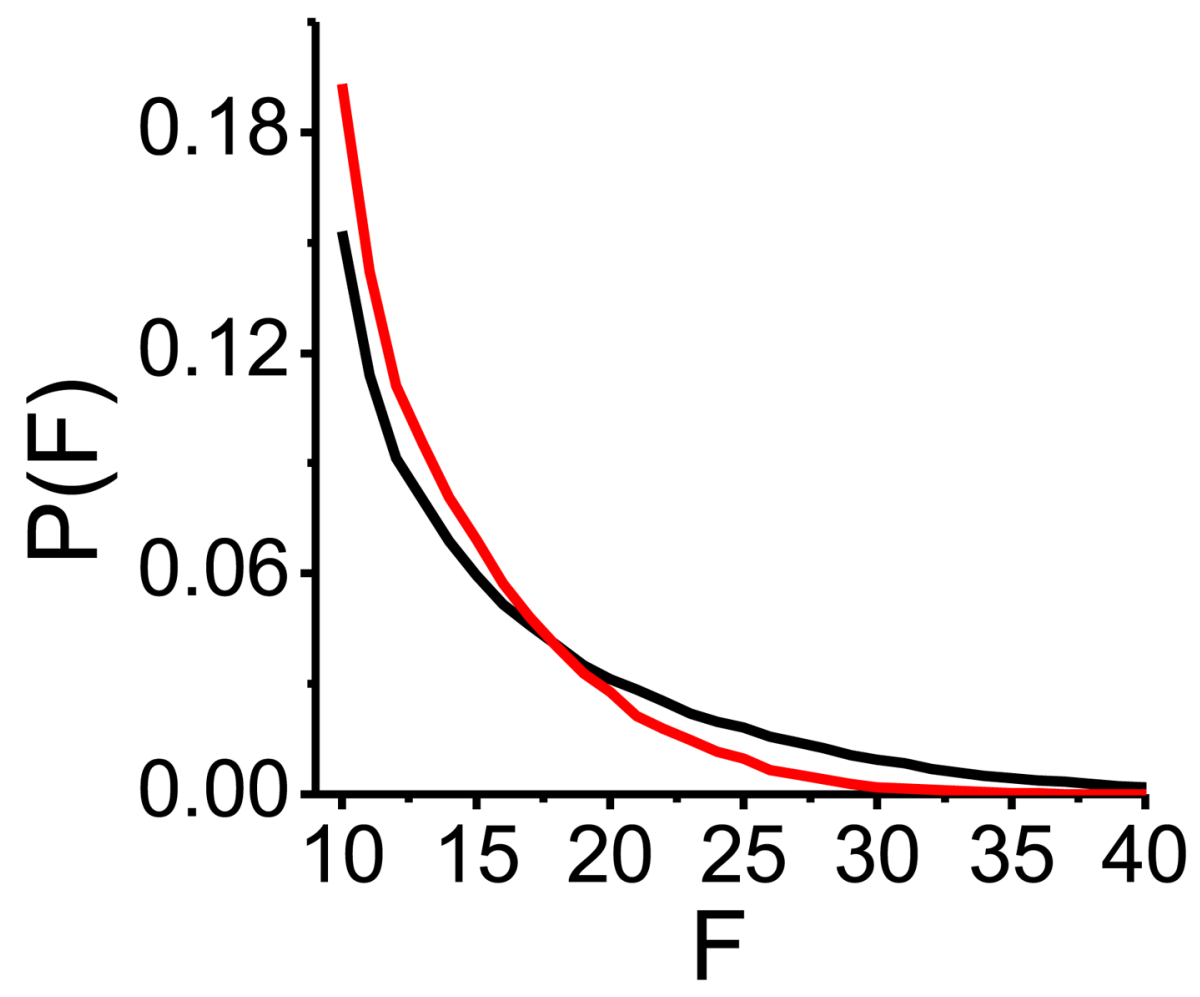

Figure 9.

FRET state dependent $P(F)$ for CaM-44-117-AF488-AF594 with CaMKII peptide in high $\mathrm{Ca}^{2+}$ buffer, as determined by cMEM. The black line shows $P(F)$ calculated in the range of $E_{\text {app }}$ from 0.0 to 0.13 corresponding to the donor only species. The red line shows $P(F)$ calculated in the $E_{a p p}$ range 0.71 to 0.91 . Both distributions were normalized so that each $P(F)$ distribution sums to one. The full two dimensional distribution is shown in Figure $7 \mathrm{~B}$. 
Table 1

Best log evidences for simulated data

\begin{tabular}{|c|c|c|c|c|}
\hline Simulation & \multicolumn{2}{|c|}{ MultiNest } & \multicolumn{2}{c|}{ cMEM } \\
\hline$\#$ & $\ln \boldsymbol{P}(\boldsymbol{D} \mid \boldsymbol{k})$ & $\boldsymbol{k}$ & $\ln \boldsymbol{P}(\boldsymbol{D} \mid \boldsymbol{n} \mathbf{L})$ & $\boldsymbol{n} \boldsymbol{L}$ \\
\hline 1 & $-2114.3 \pm 0.5$ & 2 & $-2818 \pm 4$ & 5 \\
\hline 2 & $-1883.1 \pm 0.5$ & 2 & $-2619 \pm 4$ & 5 \\
\hline 3 & $-1800.5 \pm 0.5$ & 2 & $-2560 \pm 4$ & 5 \\
\hline 4 & $-1721.0 \pm 0.4$ & 1 & $-2520 \pm 4$ & 5 \\
\hline
\end{tabular}


Table 2

Calmodulin log evidence

\begin{tabular}{|c|c|c|}
\hline Sample & MultiNest & сMEM \\
\hline CaM-44-117-AF488-AF594 high $\mathrm{Ca}^{2+}$ & $-3863.5 \pm 0.6$ & $-3369 \pm 6$ \\
\hline CaM-44-117-AF488-AF594 low $\mathrm{Ca}^{2+}$ & $-3254.9 \pm 0.6$ & $-3114 \pm 6$ \\
\hline CaM-44-117-AF488-AF594-CaMKII high $\mathrm{Ca}^{2+}$ & $-5712.9 \pm 0.6$ & $-3710 \pm 29$ \\
\hline CaM-44-117-AF488-AF594-CaMKII Apo & $-5129.5 \pm 0.7$ & $-3517 \pm 14$ \\
\hline CaM-44-117-AF488-TR high $\mathrm{Ca}^{2+}$ & $-5150.2 \pm 0.7$ & $-3836 \pm 16$ \\
\hline CaM-44-117-AF488-TR low $\mathrm{Ca}^{2+}$ & $-3541.2 \pm 0.6$ & $-3018 \pm 5$ \\
\hline CaM-44-117AF488-TR-CaMKII high $\mathrm{Ca}^{2+}$ & $-4048.6 \pm 0.6$ & $-3490 \pm 14$ \\
\hline CaM-44-117-AF488-TR-CaMKII low $\mathrm{Ca}^{2+}$ & $-2534.2 \pm 0.6$ & $-2890 \pm 7$ \\
\hline
\end{tabular}

